\title{
The review of 21 patients with antrochoanal polyp patients with antrochoanal polyp
}

\begin{abstract}
Introduction: To evaluate clinical results of patients who were treated for antrochoanal polyp in our experience.
\end{abstract}

Patients and Methods: The study included 21 patients (14 male, 7 female, mean age 27.3 \pm 16.7 , range 7-56) who had antrochoanal polyp. Axial computerized tomography (CT) in the coronal plane was performed on all patients. Endoscopic sinus surgery was performed under general or local anaesthesia.

Results: All the patients had nasal obstruction (100\%). Snoring was present in 4 patients $(19 \%)$, headache in $3(14.2 \%)$, and post-nasal drip in $6(28.5 \%)$. All the patient were treated with endoscopic endonasal surgery. There was not seen any recurrent disease.

Conclusion: AP must be considered in unilateral nasal cavity masses, particularly in young adults and children. AKP is total excision of the polyp with the antral mucosa. Endoscopic surgery is safe and effective in the treatment of AP.

Keywords: antrochoanal polyp, endoscopic surgery, nasal obstruction, antral mucosa
Volume 7 Issue 5 - 2017

\author{
Fatih Bingol,' Eda Simsek,' Buket Ozel \\ Bingol,' Gunay Kozan, ${ }^{2}$ Recai Muhammet \\ Mazlumoglu ${ }^{3}$ \\ 'Department of Otolaryngology and Head and Neck Surgery \\ Erzurum Training and Research Hospital,Turkey \\ ${ }^{2}$ Department of Otolaryngology and Head and Neck Surgery \\ Gaziantep Ersin Arslan Training and Research Hospital, Turkey \\ ${ }^{3}$ Hinis state hospital,Turkey
}

Correspondence: Fatih Bingol Department of Otolaryngology and Head and Neck Surgery Erzurum Training and Research Hospital 25070 Erzurum, Turkey, Tel +90-442-2325720, Email drfbingol@gmail.com

Received: April 03, 2017| Published: June 16, 2017
Abbreviations: CT, computerized tomography; APs, antrochoanal polyps; MRI, magnetic resonance imaging

\section{Introduction}

Antrochoanal polyps (APs) are benign lesions originating from the mucosa of the maxillary sinus and capable of extending to the choana and nasopharynx. ${ }^{1}$ Although the first case was reported by Palfyn in 1753 , it was first described in detail by Killian. ${ }^{2,3}$ APs constitute 4-6\% of adult polyps and $33 \%$ of childhood polyps. The most common symptom is unilateral nasal obstruction. Other symptoms include rhinorrhea, sinusitis and snoring. ${ }^{4}$

Treatment is possible with complete removal of the AP. As in many other sinus diseases, the essential method of treatment is endoscopic sinus surgery. ${ }^{5}$ The purpose of this study is to describe our diagnostic and therapeutic approach to AP.

\section{Patients and methods}

Twenty-one patients (14 male, 7 female, mean age $27.3 \pm 16.7$, range 7-56) undergoing surgery with a diagnosis of AP in our clinic between September 2012 and January 2015 were examined retrospectively. Detailed history was taken from all patients, and all underwent complete ear, nose and throat examination and nasal endoscopy. Computerized tomography (CT) in the coronal plane was performed on all patients (Figure 1). Endoscopic sinus surgery was performed under general or local anaesthesia. AKP was total excision of the polyp with the antral mucosa (Figure 2). Cases were monitored in terms of postsurgical complications and recurrence.

\section{Results}

AP originated from the right maxillary sinus in 12 cases, from the left maxillary sinus in eight and from the sphenoid sinus in one. Nasal obstruction was present in all cases. Snoring was present in 4 patients $(19 \%)$, headache in $3(14.2 \%)$, and post-nasal drip in $6(28.5 \%)$. Duration of symptoms ranged between 5months and 2 years. One patient had a history of previous surgery for the same reason. Paranasal CT guided both diagnosis and treatment. Ethmoid sinusitis was determined on the same side in four patients and frontal sinusitis in one. The maxillary sinus ostium was dilated in 11 of the 21 cases, and no procedures were performed on the ostium. No intra or postoperative complications developed. No recurrence was observed in any case during follow-up (duration of follow-up 5-21months).

\section{Discussion}

APs are generally seen in young adults and children. They constitute $4-6 \%$ of adult polyps and $33 \%$ of childhood polyps. ${ }^{6}$ Larsen et al. determined a mean age at diagnosis of 27 years in patients with AP. They generally originate from the maxillary sinus, but may sometimes occur in the accessory ostium. Choanal polyps have also been reported to develop in the sphenoid, posterior ethmoid and frontal sinuses. ${ }^{7}$ The etiopathogenesis of AP is unclear. Studies have reported comorbidity with chronic sinusitis and allergic rhinitis. ${ }^{8}$ It has been suggested that the maxillary sinus mucosa prolapses into the nasal cavity with negative intranasal pressure during inspiration in association with the width of the maxillary sinus. However, this theory is insufficient to account for the etiopathogenesis. Studies in the literature have implicated arachidonic acid metabolites, urokinase type plasminogen activator and plasminogen activator inhibitor-110, cytokines and IL-6 in the etiopathogenesis. ${ }^{9}$

The most common symptom is unilateral nasal obstruction. Angiofibroma, nasal glioma, inverted papilloma, mucocele mucus retention cyst, meningoencephalocele, Tornwaldt cyst and nasopharyngeal carcinoma must be considered at differential diagnosis of AP. ${ }^{10}$ There are no marked differences between AP and other polyps in terms of histology. Polyps have a pseudostratified columnar epithelium. ${ }^{11} \mathrm{CT}$ and/or magnetic resonance imaging (MRI) can be used as imaging techniques at diagnosis. APs fill the paranasal sinus at $\mathrm{CT}$ and appear as soft tissue masses extending to the central meatus via the ostia and from there to the posterior choana without causing bone destruction. They appear hypointense on T1 images at MR and hyperintense on T2 images. ${ }^{12}$ 


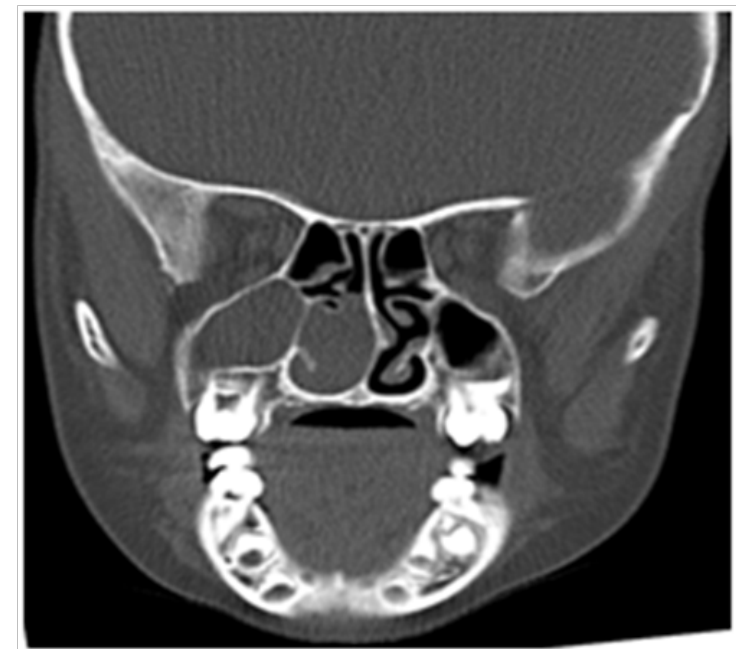

Figure I Computed tomography images, showing features consistent with an antrochoanal polyp.

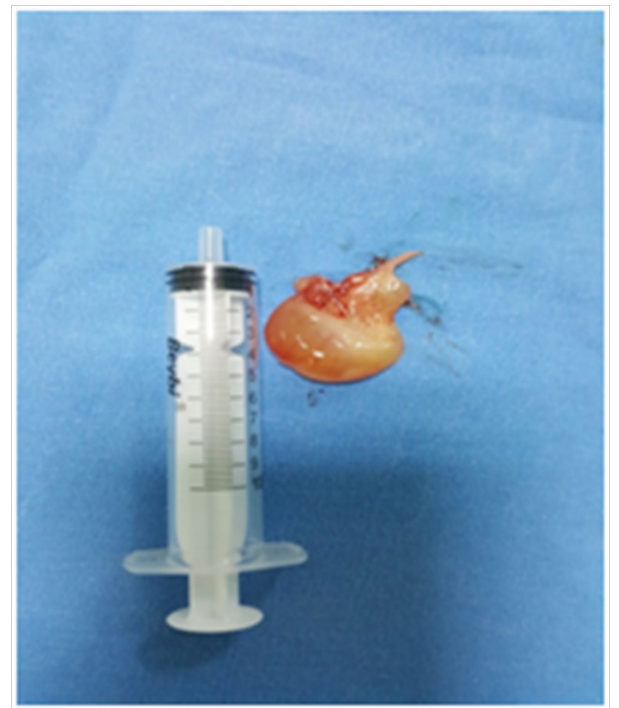

Figure 2 Macroscopic appearance of an antrochoanal polyp.

Surgery is the treatment for AP, and can be performed under general or local anaesthesia. Although the Caldwell-Luc approach provides good visualization of the antral mucosa and polyp, it also involves potential complications such as anaesthesia in the cheek, swelling and damage to developing teeth in children. ${ }^{12,13}$ Endoscopic resection is the main treatment today. Endoscopic surgery is frequently used in pathologies of the nose and sinuses since this best preserves the physiology and involves no impairment of cosmetic appearance. AP can be extracted and the osteomeatal region cleansed using the endoscopic method. ${ }^{14}$ The antral part of the polyp must be removed from where the polyp originates in order to prevent recurrence. ${ }^{9}$ Septal deviation, inferior turbinate hypertrophy and concha bullosa may also be co-present with AP, and these problems can be eliminated in the same session. ${ }^{15}$ Chronic sinusitis was present in $28.5 \%$ (6) of our cases. Endoscopic sinus surgery was performed on all patients. No intra- or postoperative complications developed. No recurrence was observed in any case during follow-up (duration of follow-up, 5-21months).

\section{Conclusion}

AP must be considered in unilateral nasal cavity masses, particularly in young adults and children. AKP is total excision of the polyp with the antral mucosa. Endoscopic surgery is safe and effective in the treatment of AP.

\section{Acknowledgments}

None.

\section{Conflicts of interest}

Author declares there are no conflicts of interest.

\section{Funding}

None.

\section{References}

1. Choudhury N, Hariri A, Saleh H. Endoscopic management of antrochoanal polyps: a single UK centre's experience. Eur Arch Otorhinolaryngol. 2015;272(9):2305-2311.

2. Palfyn J. Anatomie Chirurgicale, Paris, UK. 1753.

3. Killian G.The origin of choanal polypi. Lancet. 1906;2:81-82.

4. Hong SK, Min YG, Kim CN, et al. Endoscopic removal of the antral portion of antrochoanal polyp by powered instrumentation. Laryngoscope. 2001;111(10):1774-1778.

5. Kizil Y, Aydil U, Ceylan A, et al. Analysis of choanal polyps. J Craniofac Surg. 2014;25(3):1082-1084.

6. Porcuna DV, Gili JRM, Cabrerizo JRG, et al. Unilateral benign choanal polyp: review of 51 patients. Acta Otorrinolaringologica (English Edition). 2008;59(2):52-56.

7. Cook PR, Davis WE, McDonald R, et al. Antrochoanal polyposis: a review of 33 cases. Ear Nose Throat J. 1993;72(6):401-410.

8. Larsen K, Tos M. The estimated incidence of symptomatic nasal polyps. Acta Otolaryngol. 2002;122(2):179-182.

9. Tatlipinar A, Gökçeer T, Köksal S, et al. Our Clinical Experience In Patients With Isolated Choanal Polyps Reaching Choana. KBB-Forum. 2010;9(2):25-29.

10. Pruna X, Ibañez JM, Serres X, et al. Antrochoanal polyps in children: CT findings and differential diagnosis. Eur Radiol. 2000;10(5):849-851.

11. Chaiyasate S, Roongrotwattanasiri K, Patumanond J, et al. Antrochoanal Polyps: How Long Should Follow-Up Be after Surgery? Int $J$ Otolaryngol. 2015;2015:5.

12. Schramm VL Jr, Effron MZ. Nasal polyps in children. Laryngoscope. 1980;90(9):1488-1495.

13. Kamel R. Endoscopic transnasal surgery in antrochoanal polyps. Arch Otolaryngol Head Neck Surg. 1990;116(7):841-843.

14. Lopatin A, Bykova V, Piskunov G. Choanal polyps: one entity, one surgical approach? Rhinology. 1997;35(2):79-83.

15. De Vuysera S, Hermans R, Marchal G. Sinochoanal polypand its variant, the angiomatous polyp: MRI findings. Eur Radiol. 2001;11(1):55-58. 